\title{
2.1 Übersicht
}

Zur Existenz und Prävention von abschlusspolitischen Ergebnisspaltungen ${ }^{62}$ sind dem Verfasser der vorliegenden Arbeit die neun in der Tab. 1 angegebenen empirischen Studien bekannt. In den USA wurde die Existenz und Prävention von abschlusspolitischen Ergebnisspaltungen bereits mehrfach untersucht. In Australien und Indonesien wurde die Existenz von abschlusspolitischen Ergebnisspaltungen durch jeweils mindestens eine Studie untersucht, während die Prävention unbeachtet blieb. Dem Verfasser der vorliegenden Arbeit sind keine Studien bekannt, welche die Existenz und Prävention von abschlusspolitischen Ergebnisspaltungen für den deutschen oder europäischen Rechtsraum untersuchen. Somit adressieren die Forschungsfragen, ob (1) abschlusspolitische Ergebnisspaltungen existieren und ob (2) sie sich verhindern lassen, eine Forschungslücke im deutschen Rechtsraum. ${ }^{63}$

\begin{tabular}{lll}
\hline \multirow{2}{*}{ Land } & \multicolumn{2}{c}{ Abschlusspolitische Ergebnisspaltungen } \\
Prävention
\end{tabular}

Tab. 1: Empirische Studien zur Existenz und Prävention von abschlusspolitischen Ergebnisspaltungen nach Ländern (Quelle: Eigene Darstellung.)

Die in der Tab. 1 genannten Studien sind in der Tab. 2 chronologisch zusammengefasst. ${ }^{64}$ Im Folgenden wird untersucht, ob sich aus bestehenden Studien Erkenntnisse für die Existenz und Prävention von abschlusspolitischen Ergebnisspaltungen in Deutschland ableiten lassen. Hierfür wird zunächst der US-amerikanische und anschließend der australische und indonesische Rechtsraum betrachtet.

62 Es sei nochmals daran erinnert, dass in der vorliegenden Arbeit stets abschlusspolitische Ergebnisspaltungen mit aufgegebenen Geschäftsbereichen gemeint sind, wenn ohne nähere Spezifizierung von abschlusspolitischen Ergebnisspaltungen gesprochen wird. Siehe hierzu den Abschnitt 1.4.

63 Siehe den Abschnitt 1.3.

64 Die Tab. 2 gibt nur diejenigen Ergebnisse wieder, die für die vorliegende Arbeit relevant sind.

(C) Der/die Autor(en) 2021

K. Czupalla, Abschlusspolitische Ergebnisspaltungen mit

aufgegebenen Geschäftsbereichen nach IFRS 5, Auditing and

Accounting Studies, https://doi.org/10.1007/978-3-658-34436-8_2 


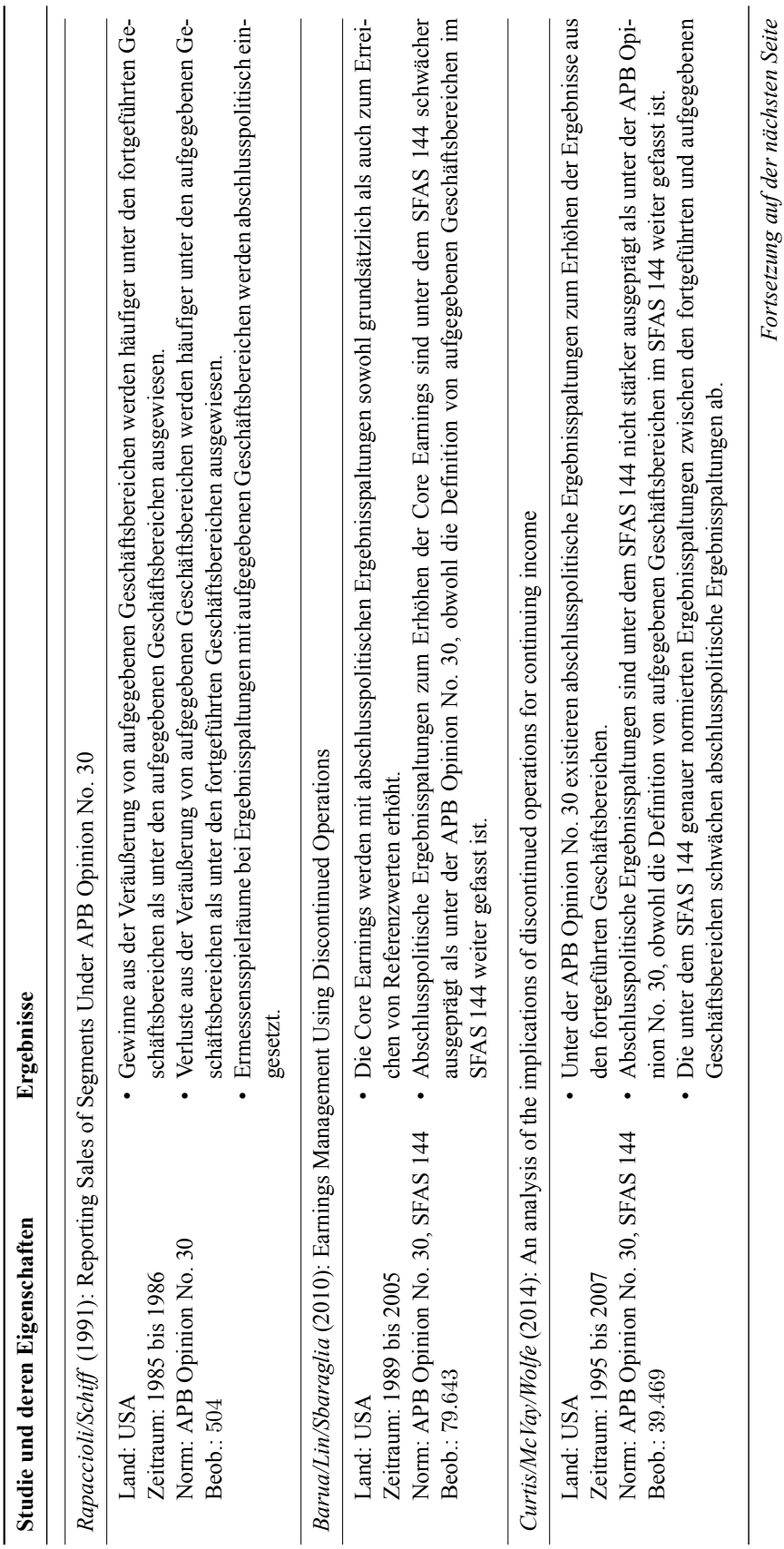




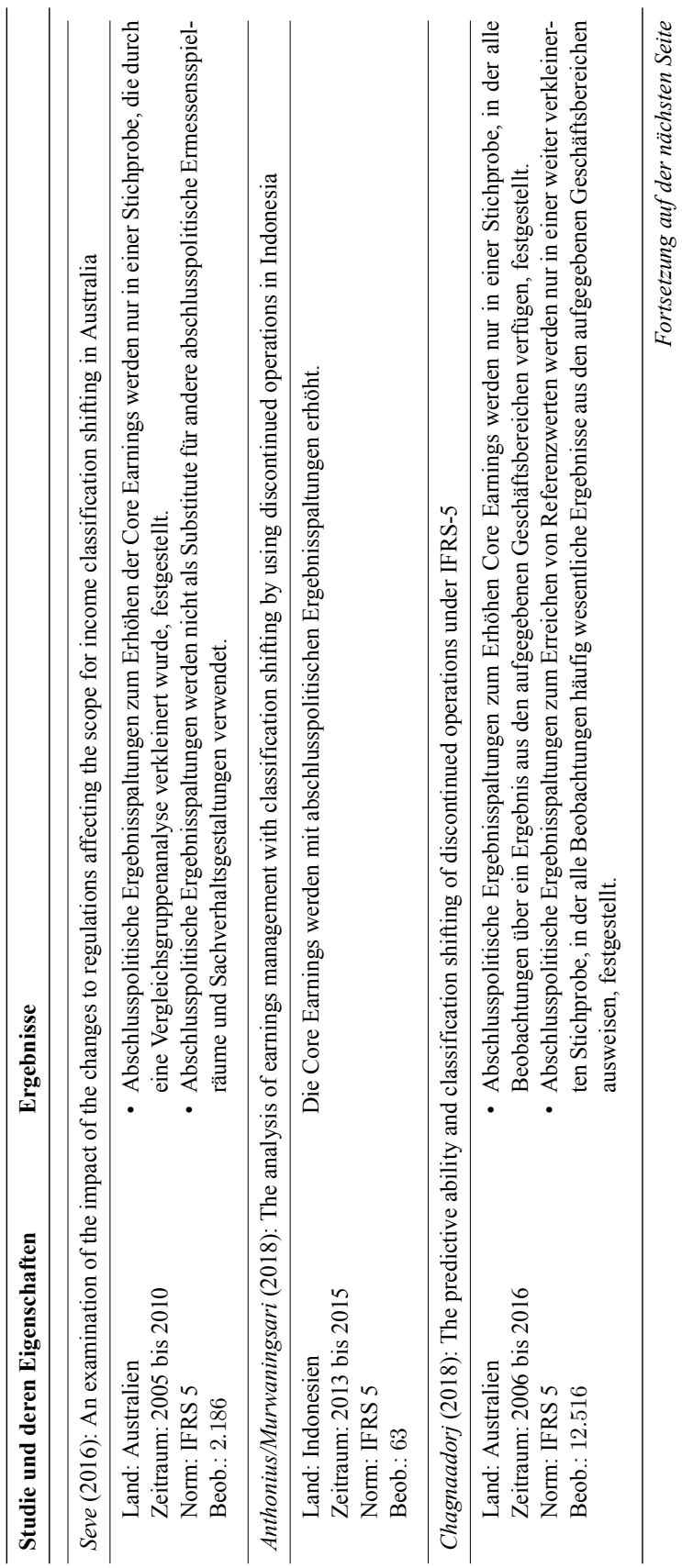




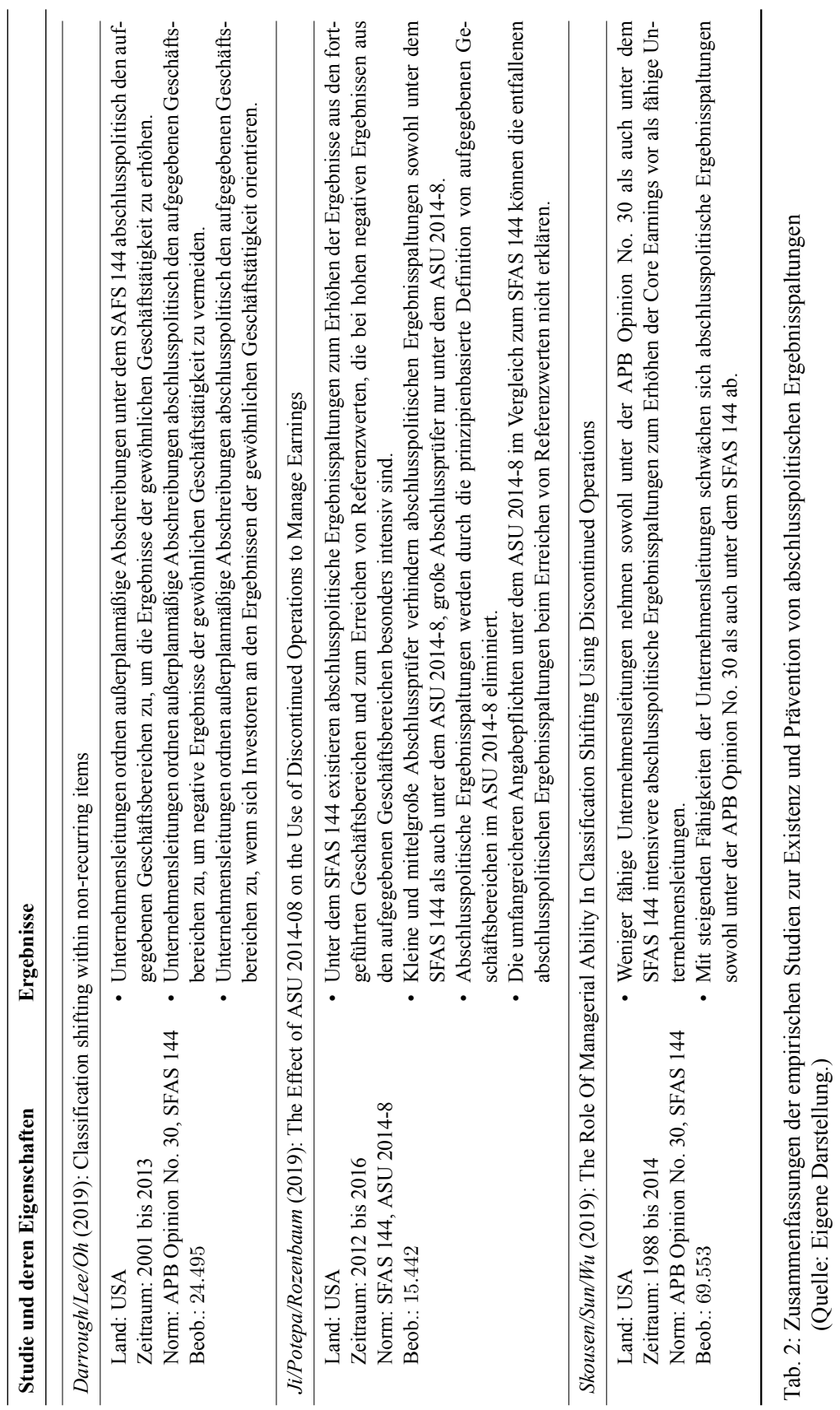




\subsection{Studien für die USA}

Rapaccioli/Schiff (1991) motivieren ihre Studie durch die unscharfe Definition von aufgegebenen Geschäftsbereichen in der von 1973 bis 2002 gültigen Accounting Principles Board Opinion No. 30 ,Reporting the Results of Operations - Reporting the Effects of Disposal of a Segment of a Business, and Extraordinary, Unusual and Infrequently Occurring Events and Transactions“ (APB Opinion No. 30), mit der Erfolge aus Verkäufen von aufgegebenen Geschäftsbereichen abschlusspolitisch entweder den fortgeführten oder den aufgegebenen Geschäftsbereichen zugeordnet werden können. Nach der Auswertung von 504 Beobachtungen aus den Jahren 1985 und 1986 stellen Rapaccioli/Schiff (1991) fest, dass Gewinne aus den Verkäufen von aufgegebenen Geschäftsbereichen signifikant häufiger in den fortgeführten Geschäftsbereichen und entsprechende Verluste signifikant häufiger in den aufgegebenen Geschäftsbereichen ausgewiesen werden. Diesen asymmetrischen Ausweis von Veräußerungserfolgen interpretieren Rapaccioli/Schiff (1991) als abschlusspolitische Ergebnisspaltungen, welche die Ergebnisse aus den fortgeführten Geschäftsbereichen erhöhen sollen. ${ }^{65}$

Die Studie von Barua/Lin/Sbaraglia (2010) umfasst rund 80.000 Beobachtungen aus den Jahren 1989 bis 2005. Barua/Lin/Sbaraglia (2010) untersuchen, ob Unternehmensleitungen Aufwendungen aus den fortgeführten Geschäftsbereichen unter den typischerweise weniger beachteten aufgegebenen Geschäftsbereichen ausweisen können. ${ }^{66}$ Tatsächlich belegen Barua/Lin/Sbaraglia (2010) für den gesamten Untersuchungszeitraum, dass Unternehmensleitungen mit abschlusspolitischen Ergebnisspaltungen die sog. „[C]ore [E]arnings “67 erhöhen. Zudem stellen Barua/Lin/Sbaraglia (2010) für den gesamten Untersuchungszeitraum fest, dass Unternehmensleitungen mit abschlusspolitischen Ergebnisspaltungen die Core Earnings erhöhen, um negative Core Earnings zu vermeiden, die Core Earnings des Vorjahres nicht zu unterschreiten oder Analystenvorhersagen zu erfüllen. Da der Untersuchungszeitraum 13 Jahre unter der in den Jahren 1973 bis 2020 gültigen APB Opinion No. 30 und vier Jahre unter der in den Jahren 2002 bis 2014 gültigen Statement of Financial Accounting Standards No. 144 ,Accounting for the Impairment or Disposal of Long-Lived Assets“ (SFAS 144) umfasst, analysieren Barua/Lin/Sbaraglia (2010) ferner, ob sich abschlusspolitische Ergebnisspaltungen unter den beiden Normen unterscheiden. Barua/Lin/Sbaraglia (2010) können abschlusspolitische Ergebnisspaltungen zum Erhöhen der Core Earnings sowohl unter der APB Opinion No. 30 als auch unter dem SFAS 144 dokumentieren. Interessanterweise sind abschlusspolitische Ergebnisspaltungen zum Erhöhen der Core Earnings unter der APB Opinion No. 30 stärker ausgeprägt, obwohl die APB Opinion No. 30 aufgegebene Geschäftsbereiche enger abgrenzt. Barua/Lin/Sbaraglia

65 Zum Vorhergehenden Rapaccioli, D./Schiff, A. (1991), S. 53-58.

66 Zum Vorhergehenden Barua, A./Lin, S./Sbaraglia, A. M. (2010), S. $1491 \mathrm{f}$.

67 Barua, A./Lin, S./Sbaraglia, A. M. (2010), S. 1491. Die Core Earnings werden in der Gleichung (6) definiert und anschließend erläutert. 
(2010) begründen die schwächeren abschlusspolitischen Ergebnisspaltungen ab dem Jahr 2002 zum einen mit der erhöhten Regulierung und Überwachung der Rechnungslegung und Abschlussprüfung durch den Sarbanes-Oxley Act, der aber dem Jahr 2002 parallel zum SFAS 144 in Kraft trat. Zum anderen lasse die weiter gefasste Definition von aufgegebenen Geschäftsbereichen unter dem SFAS 144 relativ kleine aufgegebene Geschäftsbereiche zu, denen jedoch weniger Aufwendungen abschlusspolitisch zugeordnet werden könnten. ${ }^{68}$

Curtis/McVay/Wolfe (2014) untersuchen anhand von 40.000 Beobachtungen aus den Jahren 1995 bis 2007, ob eine enger oder eine weiter gefasste Definition von aufgegebenen Geschäftsbereichen zu persistenteren Ergebnissen aus den fortgeführten Geschäftsbereichen und zu schwächeren abschlusspolitischen Ergebnisspaltungen führt. ${ }^{69}$ Hierzu vergleichen Curtis/McVay/Wolfe (2014) den Zeitraum von 1995 bis 2000 unter der APB Opinion No. 30, die eine enger gefasste Definition von aufgegebenen Geschäftsbereichen wie im IFRS 5 enthielt, mit dem Zeitraum von 2002 bis 2007 unter dem SFAS 144, der die Definition von aufgegebenen Geschäftsbereichen weiter fasste. Curtis/McVay/Wolfe (2014) zeigen, dass sich die Persistenz der Ergebnisse aus den fortgeführten Geschäftsbereichen durch SFAS 144 nicht signifikant verändert hat und abschlusspolitische Ergebnisspaltungen zum Erhöhen der Ergebnisse aus den fortgeführten Geschäftsbereichen durch SFAS 144 sogar abgeschwächt wurden. ${ }^{70}$ Schwächere abschlusspolitische Ergebnisspaltungen schließen deren Existenz jedoch nicht aus. Tatsächlich scheinen unter der APB Opinion No. 30 abschlusspolitische Ergebnisspaltungen zum Erhöhen der Ergebnisse aus den fortgeführten Geschäftsbereichen vorhanden zu sein. ${ }^{71}$ Zuletzt dokumentieren Curtis/McVay/Wolfe (2014), dass die Ergebnisse aus den fortgeführten Geschäftsbereichen durch den SFAS 144 bei Unternehmen mit einem Segment persistenter als bei Unternehmen mit mehreren Segmenten sind. Curtis/McVay/Wolfe (2014) gehen nicht davon aus, dass ihre Ergebnisse durch den im Jahr 2002 in Kraft getretenen Sarbanes-Oxley Act verfälscht sind. Vielmehr erlaube die weiter gefasste Definition von aufgegebenen Geschäftsbereichen im SFAS 144 eine feinere Ergebnisspaltung zwischen den fortgeführten und aufgegebenen Geschäftsbereichen, die bei Unternehmen mit einem Segment zu persistenteren Ergebnissen aus den fortge-

68 Zum Vorhergehenden Barua, A./Lin, S./Sbaraglia, A. M. (2010), S. 1500-1502.

69 Siehe Curtis, A./McVay, S./Wolfe, M. (2014), S. 194 f./200. Unter der Persistenz ist in diesem Kontext die Korrelation zwischen den Ergebnissen aus den fortgeführten Geschäftsbereichen in den Geschäftsjahren $t$ und $t+1$ zu verstehen. Im Abschnitt 8.5 wird auf die Persistenz von Ergebnisgrößen näher eingegangen.

70 Zum Vorhergehenden Curtis, A./McVay, S./Wolfe, M. (2014), S. 194-200.

71 Siehe den signifikant positiven Regressionskoeffizienten $\alpha_{2}=0,083$ bei Curtis, A./McVay, S./Wolfe, M. (2014), S. 198, Tab. 3. Wie im Abschnitt 9.2 analog erläutert wird, muss $\alpha_{2}$ aufgrund des Interaktionsterms $\alpha_{5}$ DiscontinuedOperations $_{t}$. RegimeShift ${ }_{t}$ so interpretiert werden, als ob die Indikatorvariable RegimeShift ${ }_{t}$ gleich null ist. Gemäß Curtis, A./McVay, S./Wolfe, M. (2014), S. 197 erfasst RegimeShift $_{t}=0$ den Zeitraum unter der APB Opinion No. 30. Da Curtis, A./McVay, S./Wolfe, M. (2014), S. 194 abschlusspolitische Ergebnisspaltungen mit positiven Korrelationen zwischen den Ergebnissen aus den aufgegebenen Geschäftsbereichen im Geschäftsjahr $t$ und den Ergebnissen aus den fortgeführten Geschäftsbereichen im Geschäftsjahr $t+1$ assoziieren, misst $\alpha_{2}$ signifikante abschlusspolitische Ergebnisspaltungen unter der APB Opinion No. 30. 
führten Geschäftsbereichen und zu schwächeren abschlusspolitischen Ergebnisspaltungen führe. $^{72}$

Darrough/Lee/Oh (2019) werten über 24.000 Beobachtungen aus den Jahren 2001 bis 2013 aus, wodurch im ersten Jahr des Untersuchungszeitraums die APB Opinion No. 30 und in den anschließenden zwölf Jahren der SFAS 144 gültig war. Darrough/Lee/Oh (2019) untersuchen, ob Unternehmensleitungen Restrukturierungsaufwendungen, außerplanmäßige Abschreibungen des Anlagevermögens und Wertminderungen von Geschäftsoder Firmenwerten, die im US-amerikanischen Rechtsraum grundsätzlich als Sonderposten innerhalb der gewöhnlichen Geschäftstätigkeit auszuweisen waren, abschlusspolitisch den aufgegebenen Geschäftsbereichen zuordnen. Den Anreiz für diese abschlusspolitischen Ergebnisspaltungen mit Sonderposten sehen Darrough/Lee/Oh (2019) in der Unternehmensbewertung durch Investoren, für welche die Ergebnisse der gewöhnlichen Geschäftstätigkeit und die darin enthaltenen Sonderposten typischerweise relevanter als die Ergebnisse aus den aufgegebenen Geschäftsbereichen seien. Folglich könne es für Unternehmensleitungen attraktiv sein, Restrukturierungsaufwendungen, außerplanmäßige Abschreibungen und Wertminderungen abschlusspolitisch den aufgegebenen Geschäftsbereichen zuzuordnen, wo sie einer hohen Unternehmensbewertung weniger abträglich sind. Darrough/Lee/Oh (2019) dokumentieren, dass außerplanmäßige Abschreibungen abschlusspolitisch den aufgegebenen Geschäftsbereichen zugeordnet werden, nicht jedoch Restrukturierungsaufwendungen und Wertminderungen von Geschäfts- oder Firmenwerten. Dass außerplanmäßige Abschreibungen abschlusspolitisch den aufgegebenen Geschäftsbereichen zugeordnet werden, können Darrough/Lee/Oh (2019) auch dann belegen, wenn sie gleichzeitig abschlusspolitische Zuordnungen von regelmäßigen betrieblichen Aufwendungen zu den aufgegebenen Geschäftsbereichen i. S. v. Barua/Lin/Sbaraglia (2010) berücksichtigen. ${ }^{73}$ Allerdings sind diese abschlusspolitischen Ergebnisspaltungen i. S. v. Barua/Lin/Sbaraglia (2010) statistisch nicht signifikant. ${ }^{74}$ Weiterführend stellen Darrough/Lee/Oh (2019) fest, dass unter der APB Opinion No. 30 keine außerplanmäßigen Abschreibungen abschlusspolitisch den aufgegebenen Geschäftsbereichen zugeordnet werden, sondern diese abschlusspolitischen Ergebnisspaltungen erst durch den SFAS 144 einsetzen. ${ }^{75}$

Darrough/Lee/Oh (2019) vermuten zudem ausgeprägtere abschlusspolitische Zuordnungen von außerplanmäßigen Abschreibungen zu den aufgegebenen Geschäftsbereichen, wenn Unternehmensleitungen nichtnegative Ergebnisse der gewöhnlichen Geschäftstätigkeit oder Ergebnisse der gewöhnlichen Geschäftstätigkeit mindestens in der Höhe der Vorjahre erzielen. Allerdings ergeben sich nur sehr schwache Anzeichen, dass Unterneh-

72 Zum Vorhergehenden Curtis, A./McVay, S./Wolfe, M. (2014), S. 194 f./200.

73 Zum Vorhergehenden Darrough, M./Lee, Y. G./Oh, H. I. (2019), S. 185-190/194-196/199 f./203, Fn. 28.

74 Siehe den statistisch nicht signifikant negativen Regressionskoeffizienten $\varphi$ in Höhe von $-0,1921$ bei Darrough, M./Lee, Y. G./Oh, H. I. (2019), Tab. 8.

75 So Darrough, M./Lee, Y. G./Oh, H. I. (2019), S. 196/203, Fn. 28. 
mensleitungen außerplanmäßige Abschreibungen abschlusspolitisch den aufgegebenen Geschäftsbereichen zuordnen, wenn sie negative Ergebnisse der gewöhnlichen Geschäftstätigkeit vermeiden wollen. Dagegen können Darrough/Lee/Oh (2019) beim Erreichen oder Übertreffen der Vorjahresergebnisse der gewöhnlichen Geschäftstätigkeit keine abschlusspolitischen Ergebnisspaltungen mit außerplanmäßigen Abschreibungen nachweisen. Abschließend zeigen Darrough/Lee/Oh (2019), dass die Unternehmensleitungen von Unternehmen, deren Bewertungen im Vergleich zu anderen Unternehmen stärker von den Ergebnissen der gewöhnlichen Geschäftstätigkeit als von den Jahresergebnissen abhängen, außerplanmäßige Abschreibungen abschlusspolitisch den aufgegebenen Geschäftsbereichen zuordnen. ${ }^{76}$

Ji/Potepa/Rozenbaum (2019) untersuchen anhand von über 15.000 Beobachtungen aus dem Zeitraum von 2012 bis 2016 die Frage, ob abschlusspolitische Ergebnisspaltungen durch das seit dem Jahr 2014 gültige Accounting Standards Update No. 2014-8 „Reporting Discontinued Operations and Disclosures of Disposals of Components of an Entity" (ASU 2014-8) zu- oder abgenommen haben. Hierzu lassen Ji/Potepa/Rozenbaum (2019) das Übergangsjahr 2014 unberücksichtigt und vergleichen den Zeitraum von 2012 bis 2013 unter dem SFAS 144 mit dem Zeitraum von 2015 bis 2016 unter dem ASU 2014-8. Ji/Potepa/Rozenbaum (2019) dokumentieren zunächst abschlusspolitische Ergebnisspaltungen zum Erhöhen der Ergebnisse der gewöhnlichen Geschäftstätigkeit unter dem SFAS 144, die durch das ASU 2014-8 deutlich abgenommen haben. Anschließend untersuchen Ji/Potepa/Rozenbaum (2019), ob abschlusspolitische Ergebnisspaltungen beim Erreichen von Referenzwerten unter dem SFAS 144 existieren und durch das ASU 2014-8 zu- oder abgenommen haben. Ji/Potepa/Rozenbaum (2019) können weder unter dem SFAS 144 noch unter dem ASU 2014-8 abschlusspolitische Ergebnisspaltungen beim Erreichen von nichtnegativen Ergebnissen der gewöhnlichen Geschäftstätigkeit belegen. Dagegen berichten Ji/Potepa/Rozenbaum (2019), dass abschlusspolitische Ergebnisspaltungen unter dem SFAS 144 beim Erreichen oder Übertreffen der Vorjahresergebnisse der gewöhnlichen Geschäftstätigkeit bzw. von Analystenvorhersagen existieren, aber unter dem ASU 2014-8 nicht mehr vorhanden sind. Ji/Potepa/Rozenbaum (2019) präzisieren, dass abschlusspolitische Ergebnisspaltungen beim Erreichen oder Übertreffen von Analystenvorhersagen vor allem bei hohen negativen Ergebnissen aus den aufgegebenen Geschäftsbereichen unter dem SFAS 144 vorgenommen werden. Die umfangreicheren Angabepflichten unter dem ASU 2014-8 können die entfallenen abschlusspolitischen Ergebnisspaltungen beim Erreichen oder Übertreffen von Analystenvorhersagen jedoch nicht erklären, da bereits unter dem SAFS 144 freiwillig Angaben in vergleichbarem Umfang gemacht werden. ${ }^{77}$

76 Zum Vorhergehenden Darrough, M./Lee, Y. G./Oh, H. I. (2019), S. 188/197-199.

77 Zum Vorhergehenden Ji, Y./Potepa, J./Rozenbaum, O. (2019), S. 4-8/13-19/29/34-39. 
Ji/Potepa/Rozenbaum (2019) stellen bezüglich der Prävention von abschlusspolitischen Ergebnisspaltungen fest, dass kleine und mittelgroße Abschlussprüfer abschlusspolitische Ergebnisspaltungen unter dem SFAS 144 und dem ASU 2014-8 beim Erreichen oder Übertreffen von Analystenvorhersagen verhindern können, wohingegen große Abschlussprüfer hierbei nur unter dem ASU 2014-8 erfolgreich sind. Zudem zeigen Ji/Potepa/Rozenbaum (2019), dass positive Ergebnisse aus den aufgegebenen Geschäftsbereichen weder unter dem SFAS 144 noch unter dem ASU 2014-8 persistent sind, während negative Ergebnisse aus den aufgegebenen Geschäftsbereichen unter dem SAFS 144 persistent sind. Diese asymmetrische Persistenz interpretieren Ji/Potepa/Rozenbaum (2019) als abschlusspolitische Ergebnisspaltungen, die nur bei negativen Ergebnissen aus den aufgegebenen Geschäftsbereichen auftreten, aber durch den ASU 2014-8 eliminiert werden. Insgesamt führen Ji/Potepa/Rozenbaum (2019) die entfallenen abschlusspolitischen Ergebnisspaltungen unter dem ASU 2018-4 auf dessen stärkere Prinzipienbasierung zurück, die Umgehungsmöglichkeiten einschränke sowie die diesbezüglichen Prüfungsergebnisse von Regulierungsbehörden und Abschlussprüfern weniger vorhersehbar gestalte. ${ }^{78}$

Skousen/Sun/Wu (2019) werten rund 70.000 Beobachtungen aus dem Zeitraum von 1988 bis 2014 aus, wovon die ersten 14 Jahre auf die APB Opinion No. 30 und die darauffolgenden 13 Jahre auf den SFAS 144 entfallen. Skousen/Sun/Wu (2019) motivieren ihre Studie durch die Frage, ob fähige Unternehmensleitungen schwächere abschlusspolitische Ergebnisspaltungen vornehmen als weniger fähige Unternehmensleitungen. ${ }^{79}$ Skousen $/ \mathrm{Sun} / W u$ (2019) messen die Fähigkeiten von Unternehmensleitungen unter Rückgriff auf Demerjian/Lev/McVay (2012), welche eine Messgröße für die Fähigkeit von Unternehmensleitungen mit einem mehrstufigen, komplexen Verfahren berechnen. ${ }^{80}$ Vereinfacht gesagt berechnen Demerjian/Lev/McVay (2012) zunächst, wie viel die materiellen und immateriellen Produktionsmittel kosten, mit denen Unternehmen ihre Umsätze generieren. Anschließend werden diese Kosten-Nutzen-Verhältnisse der einzelnen Unternehmen in Relation zum Unternehmen mit dem besten Kosten-Nutzen-Verhältnis innerhalb einer Branche gesetzt, woraus sich eine Messgröße für die relative Unternehmenseffizienz ergibt. Zuletzt wird die Messgröße für die Fähigkeiten der Unternehmensleitungen aus der Messgröße für die relative Unternehmenseffizienz extrahiert, indem Eigenschaften wie die Unternehmensgröße und das Unternehmensalter, welche die Unternehmenseffizienz beeinflussen können, aber von Unternehmensleitungen zumindest kurzfristig nicht veränderbar sind, konstant gehalten werden. ${ }^{81}$ Skousen/Sun/Wu (2019) verdichten diese intervallskalierte Messgröße für die Fähigkeiten der Unternehmensleitungen von Demerjian/Lev/McVay (2012) auf eine ordinalskalierte Messgröße. ${ }^{82}$ Diese Messgröße kann Werte zwischen 0 und 1 annehmen,

78 Zum Vorhergehenden Ji, Y./Potepa, J./Rozenbaum, O. (2019), S. 3/7/20-22/25 f./40 f.

79 Zum Vorhergehenden Skousen, C./Sun, L./Wu, K. (2019), S. 114/118.

80 Siehe Skousen, C./Sun, L./Wu, K. (2019), S. 131 unter Verweis auf Demerjian, P./Lev, B./McVay, S. (2012), S. 1232-1237.

81 Zum Vorhergehenden Demerjian, P./Lev, B./McVay, S. (2012), S. 1232-1237.

82 Vgl. Demerjian, P./Lev, B./McVay, S. (2012), S. 1237 f.; Skousen, C./Sun, L./Wu, K. (2019), S. 121/131. 
die extrem unfähige respektive extrem fähige Unternehmensleitungen signalisieren. Der Mittelwert in Höhe von 0,564 markiert eine durchschnittlich fähige Unternehmensleitung. Skousen/Sun/Wu (2019) dokumentieren, dass weniger fähige Unternehmensleitungen abschlusspolitische Ergebnisspaltungen sowohl unter der APB Opinion No. 30 als auch unter dem SFAS 144 vornehmen. Bezüglich der Prävention von abschlusspolitischen Ergebnisspaltung stellen Skousen/Sun/Wu (2019) fest, dass sich unter beiden Normen mit steigenden Fähigkeiten der Unternehmensleitungen die abschlusspolitischen Ergebnisspaltungen abschwächen. ${ }^{83}$

\subsection{Zwischenfazit}

Als Zwischenfazit ist festzuhalten, dass alle Studien abschlusspolitische Ergebnisspaltungen unter der APB Opinion No. 30, dem SFAS 144 oder dem ASU 2014-8 dokumentieren. ${ }^{84}$ Unter der APB Opinion No. 30, die dem IFRS 5 am ähnlichsten ist, ${ }^{85}$ belegen Rapaccioli/Schiff (1991), Barua/Lin/Sbaraglia (2010), Curtis/McVay/Wolfe (2014) und Skousen/Sun/Wu (2019) abschlusspolitische Ergebnisspaltungen. ${ }^{86}$ Barua/Lin/Sbaraglia (2010) und Curtis/McVay/Wolfe (2014) berichten unter der APB Opinion No. 30 sogar intensivere abschlusspolitische Ergebnisspaltungen als unter dem SFAS $144 .{ }^{87}$ Lediglich Darrough/Lee/Oh (2019) können unter der APB Opinion No. 30 keine abschlusspolitischen Ergebnisspaltungen nachweisen. Allerdings könnte dieser fehlende Nachweis durch den Untersuchungszeitraum begründet sein, von dem zwölf Jahre auf den SFAS 144 und nur ein Jahr auf die APB Opinion No. 30 entfallen. ${ }^{88}$ Als Motive für abschlusspolitische Ergebnisspaltungen unter der APB Opinion No. 30 dokumentieren Rapaccioli/Schiff (1991) und Curtis/McVay/Wolfe (2014) ein Erhöhen der Ergebnisse aus den fortgeführten Geschäftsbereichen, Barua/Lin/Sbaraglia (2010) ein Erhöhen der Core Earnings sowie Skousen/Sun/Wu (2019) ein Erhöhen der Core Earnings durch weniger fähige Unternehmensleitungen. ${ }^{89}$ Bezüglich der Prävention von abschlusspolitischen Ergebnisspaltungen zeigen Skousen/Sun/Wu (2019), dass sich unter der APB Opinion No. 30 abschlusspolitische Ergebnisspaltungen mit steigenden Fähigkeiten der Unternehmensleitungen abschwä-

83 Zum Vorhergehenden Skousen, C./Sun, L./Wu, K. (2019), S. 121/127 f./131.

84 Vgl. Barua, A./Lin, S./Sbaraglia, A. M. (2010), S. 1502; Curtis, A./McVay, S./Wolfe, M. (2014), S. 194-198; Darrough, M./Lee, Y. G./Oh, H. I. (2019), S. 194-196; Ji, Y./Potepa, J./Rozenbaum, O. (2019), S. 15/18; Rapaccioli, D./Schiff, A. (1991), S. 53 f./58; Skousen, C./Sun, L./Wu, K. (2019), S. $127 \mathrm{f}$.

85 Siehe den Unterabschnitt 4.1.4.

86 Siehe Barua, A./Lin, S./Sbaraglia, A. M. (2010), S. 1502; Curtis, A./McVay, S./Wolfe, M. (2014), S. 194-198; Rapaccioli, D./Schiff, A. (1991), S. 53 f./58; Skousen, C./Sun, L./Wu, K. (2019), S. 127 f.

87 Siehe Barua, A./Lin, S./Sbaraglia, A. M. (2010), S. 1502; Curtis, A./McVay, S./Wolfe, M. (2014), S. $198 / 200$.

88 Zum Vorhergehenden Darrough, M./Lee, Y. G./Oh, H. I. (2019), S. 186/188/190/196/203, Fn. 28.

89 Zum ersten Motiv Curtis, A./McVay, S./Wolfe, M. (2014), S. 194-198; Rapaccioli, D./Schiff, A. (1991), S. 53 f./58. Zum zweiten Motiv Barua, A./Lin, S./Sbaraglia, A. M. (2010), S. 1502. Zum dritten Motiv Skousen, C./Sun, L./Wu, K. (2019), S. 127 f. 
chen. ${ }^{90}$ Ji/Potepa/Rozenbaum (2019) berichten, dass kleine und mittelgroße Abschlussprüfer abschlusspolitischen Ergebnisspaltungen sowohl unter dem SFAS 144 als auch unter dem ASU 2014-8 verhindern, während großen Abschlussprüfer hierbei nur unter dem ASU 2014-8 erfolgreich sind..$^{91}$ Nach den Studien aus den USA werden nun die Studien aus Australien und Indonesien betrachtet. Da diese den IFRS 5 untersuchen, ${ }^{92}$ lassen sie sich tendenziell leichter auf Deutschland übertragen.

\subsection{Studien für Australien und Indonesien}

Seve (2016) untersucht, ob unter dem im Jahr 2005 eingeführten Australian Accounting Standards Board Standard 5 ,Noncurrent Assets Held for Sales and Discontinued Operations“ (AASB 5), der bis auf wenige regionale Ergänzungen den IFRS 5 wiedergibt, abschlusspolitische Ergebnisspaltungen existieren und andere abschlusspolitische Maßnahmen abgelöst haben. Während Seve (2016) in einer Stichprobe mit über 2.000 Beobachtungen aus den Jahren 2005 bis 2010 abschlusspolitische Ergebnisspaltungen zum Erhöhen der Core Earnings nicht nachweisen kann, gelingt ihm dieser Nachweis in einer Vergleichsgruppenanalyse (propensity score matching) mit rund 1.000 Beobachtungen. Mit der Vergleichsgruppenanalyse ermittelt Seve (2016) zu jeder Beobachtung mit aufgegebenen Geschäftsbereichen mindestens eine Beobachtung ohne aufgegebene Geschäftsbereiche, die hinsichtlich diverser Eigenschaften wie der Unternehmensgröße und dem Verschuldungsgrad zur Beobachtung mit aufgegebenen Geschäftsbereichen vergleichbar ist. Die so gebildeten Vergleichsgruppen sollen systematische Unterschiede zwischen den Beobachtungen mit und ohne aufgegebenen Geschäftsbereichen verhindern, die abschlusspolitische Ergebnisspaltungen überdecken oder irrtümlich suggerieren können. Zurückkehrend zur vollständigen Stichprobe dokumentiert Seve (2016), dass unter dem AASB 5 sowohl Ermessensspielräume in Form von diskretionären Periodenabgrenzungen als auch Sachverhaltsgestaltungen in Form von beschleunigten Umsatzakten sowie gekürzten Verwaltungs-, Werbe-, Forschungs- und Entwicklungsaufwendungen nicht durch Ermessensspielräume in Form von abschlusspolitischen Ergebnisspaltungen ersetzt werden. ${ }^{93}$

Chagnaadorj (2018) untersucht in ihrer ebenfalls australischen Studie, ob unter IFRS 5 abschlusspolitische Ergebnisspaltungen zum Erhöhen der Core Earnings und zum Erreichen von Referenzwerten existieren. Die Studie von Seve (2016) scheint Chagnaadorj (2018) nicht zu kennen. Chagnaadorj (2018) kann anhand ihrer vollständigen Stichprobe mit rund 13.000 Beobachtungen aus den Jahren 2006 bis 2016 keine abschlusspolitischen Ergebnis-

90 So Skousen, C./Sun, L./Wu, K. (2019), S. 127 f.

91 Siehe Ji, Y./Potepa, J./Rozenbaum, O. (2019), S. 40.

92 Vgl. Anthonius/Murwaningsari, E. (2018), S. 109; Chagnaadorj, O. (2018), S. 3 f.; Seve, F. (2016), S. 82-84.

93 Zum Vorgergehenden Seve, F. (2016), S. 24/82-84/101 f./126 f./154-157/210-213/243-249/255 f./260262. Auf Sachverhaltsgestaltungen wird im Unterabschnitt 4.2.2 und auf diskretionäre Periodenabgrenzungen wird im Abschnitt 8.5 eingegangen. 
spaltungen zum Erhöhen der Core Earnings belegen. Allerdings dokumentiert Chagnaadorj (2018) mit einer verkleinerten Stichprobe, die 1.625 Beobachtungen mit Ergebnissen aus den aufgegebenen Geschäftsbereichen enthält, abschlusspolitische Ergebnisspaltungen zum Erhöhen der Core Earnings. Dagegen kann Chagnaadorj (2018) mit dieser verkleinerten Stichprobe keine abschlusspolitischen Ergebnisspaltungen zum Erreichen von nichtnegativen Ergebnissen aus den fortgeführten Geschäftsbereichen oder zum Erreichen von Ergebnissen aus den fortgeführten Geschäftsbereichen über den Vorjahreswerten nachweisen. Mit einer weiter verkleinerten Stichprobe, deren 845 Beobachtungen in mindestens zwei aufeinanderfolgenden Geschäftsjahren wesentliche Ergebnisse aus den aufgegebenen Geschäftsbereichen ausweisen, kann Chagnaadorj (2018) abschlusspolitische Ergebnisspaltungen sowohl zum Erhöhen der Core Earnings als auch zum Erreichen von nichtnegativen Ergebnissen aus den fortgeführten Geschäftsbereichen und von Ergebnissen aus den fortgeführten Geschäftsbereichen über den Vorjahreswerten zeigen. ${ }^{94}$

Anthonius/Murwaningsari (2018) untersuchen abschlusspolitische Ergebnisspaltungen von indonesischen Unternehmen. In einer Stichprobe mit 63 Beobachtungen aus den Jahren 2013 bis 2015 dokumentieren Anthonius/Murwaningsari (2018) abschlusspolitische Ergebnisspaltungen zum Erhöhen der Core Earnings. Aufgrund der kleinen Stichprobe schränken Anthonius/Murwaningsari (2018) die Aussagekraft ihrer Studie jedoch ein. ${ }^{95}$

\subsection{Zwischenfazit}

Als Zwischenfazit ist festzuhalten, dass alle Studien für den australischen und indonesischen Rechtsraum abschlusspolitische Ergebnisspaltungen zum Erhöhen der Core Earnings unter dem dort gültigen IFRS 5 nachweisen. ${ }^{96}$ Allerdings gelingt dieser Nachweis den beiden australischen Studien nur mit einer Vergleichsgruppenanalyse oder mit einer Stichprobe, die ausschließlich Beobachtungen mit aufgegebenen Geschäftsbereichen enthält. ${ }^{97}$ Die Aussagekraft der indonesischen Studie ist aufgrund ihrer sehr kleinen Stichprobe eingeschränkt. ${ }^{98}$ Die Prävention einer abschlusspolitischen Ergebnisspaltung wird in keiner der drei Studien zu IFRS 5 untersucht.

\subsection{Gesamtfazit}

Als Gesamtfazit ist festzuhalten, dass abschlusspolitische Ergebnisspaltungen fast immer belegt werden können. Während abschlusspolitische Ergebnisspaltungen unter der USamerikanischen APB Opinion No. 30, die dem IFRS 5 ähnelt, relativ leicht nachgewie-

94 Zum Vorhergehenden Chagnaadorj, O. (2018), S. 3 f./24/61-66/70-73.

95 Zum Vorhergehenden Anthonius/Murwaningsari, E. (2018), S. 109-116.

96 Siehe Anthonius/Murwaningsari, E. (2018), S. 111/114-116; Chagnaadorj, O. (2018), S. 61/63/70-73; Seve, F. (2016), S. 210-213/243/260.

97 So Chagnaadorj, O. (2018), S. 61/63/70-73; Seve, F. (2016), S. 210-213/243/260.

98 Vgl. Anthonius/Murwaningsari, E. (2018), S. 111. 
sen werden können, ${ }^{99}$ gelingt dieser Nachweis unter dem IFRS 5 in Indonesien nur mit einer sehr kleinen Stichprobe und in Australien nur unter restriktiven Voraussetzungen. ${ }^{100}$ Zur Prävention von abschlusspolitischen Ergebnisspaltungen unter der US-amerikanischen APB Opinion No. 30 wurden nur die Fähigkeiten der Unternehmensleitungen untersucht und dokumentiert, dass sich abschlusspolitische Ergebnisspaltungen mit zunehmenden Fähigkeiten der Unternehmensleitungen abschwächen. ${ }^{101}$ Unter dem IFRS 5 wurde die Prävention von abschlusspolitischen Ergebnisspaltungen bislang nicht untersucht.

Dieses Kapitel wird unter der Creative Commons Namensnennung 4.0 International Lizenz (http://creativecommons.org/licenses/by/4.0/deed.de) veröffentlicht.

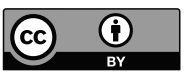

99 Siehe Barua, A./Lin, S./Sbaraglia, A. M. (2010), S. 1502; Curtis, A./McVay, S./Wolfe, M. (2014), S. 194-198; Rapaccioli, D./Schiff, A. (1991), S. 53 f./58; Skousen, C./Sun, L./Wu, K. (2019), S. 127 f.

100 Vgl. Chagnaadorj, O. (2018), S. 61/63/70-73; Seve, F. (2016), S. 210-213/243/260.

101 Siehe Skousen, C./Sun, L./Wu, K. (2019), S. 127 f. 